\title{
Tactile Imaging and Distributed Strain Sensing in Highly Flexible Carbon Nanofiber/Polyurethane Nanocomposites
}

\author{
T. N. Tallman ${ }^{1 *}$, S. Gungor ${ }^{2}$, K. W. Wang ${ }^{1}$, and C. E. Bakis ${ }^{2}$ \\ ${ }^{1}$ Department of Mechanical Engineering, University of Michigan, Ann Arbor, Michigan 48109 \\ ${ }^{2}$ Department of Engineering Science and Mechanics, Pennsylvania State University, University \\ Park, Pennsylvania 16802
}

\begin{abstract}
Highly flexible nanocomposites have tremendous potential as smart, self-sensing materials because their conductivity is inherently linked to their mechanical state. Herein, carbon nanofiber $(\mathrm{CNF})$ /polyurethane (PU)nanocomposites are studiedfor tactile imaging and distributed strain sensing via electrical impedance tomography (EIT) by investigatingthe influence of filler volume fraction on microscale morphology, piezoresistive response while bonded to mechanically loaded substrates, and sensitivity to distributed strain.Load testing of the bonded sensor reveals that viscoelasticity and filler volume fraction profoundly affect the piezoresistive response. EIT is able to accurately capture and discern between multiple points of contact in each volume fraction with lower volume fractions being more sensitivethereby demonstrating the potential of utilizing tomographic methods for tactile imaging and distributed strain sensing in PU-based nanocomposites.
\end{abstract}

\section{Introduction}

Highly flexible skins capable of tactile imaging and distributed strain sensing are of keen interest to robotic and biomedical applications wherein it is necessary to spatially resolve points of contact [1]. For example, spatially resolving points of contact with a prosthetic via a sensing skin could be used to provide haptic feedback to the wearer. Additionally, robotic grappling

\footnotetext{
*Corresponding author. E-mail: ttallman@umich.edu (Tyler N. Tallman)

Telephone: 734-615-8747

(C) 2015. This manuscript version is made available under the Elsevier user license http://www.elsevier.com/open-access/userlicense/1.0/
} 
mechanisms in surgical venues would improve if they were capable of sensing when they have made contact. In such an application the sensing skin would need to be compliant enough to accommodate the robot's motion. The development of these skins and the detection of contact with them, however, pose challenges in the areas of material development, sensor deployment, and image processing. Locating contact within flexible planar skins has been approached by incorporating a sensing medium into a compliant matrix such that a grid is formed either by the sensing medium [2] or by line electrodes sandwiching the sensing medium [3]. Pressure-induced capacitance changes are then measured at the grid points so that pressure fields can be imaged by interpolating capacitance changes between measurement points.Despite the success of this approach, an important limitation is the dependence on a grid of sensors. That is, sensitivity to stimuli may be lost away from grid points particularly in a sparse grid. This can be circumvented by increasing the density of the grid, but this will necessarily add to both the manufacturing complexity andcost while simultaneously increasing the number of measurements necessary to form an image. Sparse grids, furthermore, are not particularly adept at discerning the geometry of pressure-induced strain fields.

Nanocomposites have tremendous potential as an alternative to embedded grid systems for flexible skins capable of tactile imaging and distributed strain sensing. Key to this potential is piezoresistivity. Piezoresistivity is rooted in the dependence of nanocomposite conductivity on well-connected nanofiller networks through which electrons traverse by tunneling between sufficiently proximate nanofillers. Mechanical influences such as applied forces, displacements, or pressures strain the nanocomposite and the nanofillers consequently reorient thereby altering their proximity. Because the equivalent resistance felt by a tunneling electron depends exponentially on the length of the tunneling junction [4], even slight strains can markedly affect conductivity. Locating strain-induced conductivity changes is therefore tantamount to locating the mechanical perturbation causing strain.

Considerable effort has been dedicated to researching nanocomposites as high-sensitivity strain sensors [5] [6] [7] [8] [9] [10] [11] and for integrated damage detection [12] [13] [14] [15] [16] [17] [18] [19] [20] [21] [22] [23] [24] [25] [26].Piezoresistive studies specifically concerned with carbon nanofibers (CNFs) have also been conducted[27] [28]. Methods of locating strain or 
damage within a nanocomposite plane can be broadly categorized in two ways - interpolation of resistance changes [17] [18] [19] [20] or tomographic methods [21] [22] [23] [24] [25] [26]. The former is similar to the aforementioned grid approaches except that resistance is measured instead of capacitance. It also, however, suffers from the same limitations. Conversely, tomographic methods such as electrical impedance tomography (EIT) require fewer measurement pointsor electrodes located only along the periphery of the material and can discern the geometry of the strain field. EIT is more computationally expensive, but because of the rapid growth of computing power, this is a relative assessment. EIT has also been studied for pressure detection in conductive fabrics [29] [30] [31].

In light of the preceding discussion, we identify a novelapproach for advancing the state of the art - employing EIT for tactile imaging and distributed strain sensing in highly flexible nanocomposites. This approach combines the advantages of EIT (superior images and no need to build sensor grids into the material) with the pervasiveness of a nanofiller networkacting as an integrated sensor network thereby ensuring sensitivity throughout the material.In addition to the aforementioned advantages of nanocomposite skins for tactile imaging, they are also much less complex and costly to manufacture. Weherein explore the influence of filler volume fraction on the microscale morphology, viscoelastic piezoresistive response while bonded to a mechanically loaded substrate, and sensitivity to distributed strain in highly flexible CNF/polyurethane (PU) nanocomposites. The influence of filler volume fraction is an important consideration because an ideal nanocomposite material for tactile imaging will be highly sensitive with well-dispersed fillers. That is, the material should produce large conductivity changes under mechanical loading and the nanofiller network should be equally distributed throughout the composite. These qualities, however, are generally at odds. Filler volume fractions just past the percolation threshold are generally more sensitive to strain, but low filler loading may lead to regions with insufficiently many nanofillers to achieve percolation. This manuscript is an exploratory investigation into the potential of a low-cost and easy to manufacture CNF/PU nanocomposite for tactile sensing via EIT.

The remainder of this investigation is organized as follows. First, the EIT formulation is presented. Next, our experimental procedures regarding CNF/PU manufacturing, bonded tensile 
testing, and EIT are detailed. Our results are then presented with a discussion. And, finally, we end with a brief summary and conclusions.

\section{Electrical Impedance Tomography}

EIT is a tomographic method of imaging an internal conductivity distribution. Procedurally, the domain to be imaged is lined with electrodes as shown in Figure 1. Measurements proceed by injecting current between the first electrode pair and recording the voltage between electrode pairs not actively involved in the current injection. The current injection is then moved around the domain such that every electrode pair receives an injection while the boundary voltages are continually collected.

Computationally, EIT can be thought of a minimization problem requiring the solution of an underdetermined inverse problem. That is, it endeavors to minimize the difference between experimentally measured boundary voltages and an analytical simulation that predicts the same boundary voltages as a function of the conductivity distribution. These two components of EIT are referred to as the forward problem and the inverse problem. 


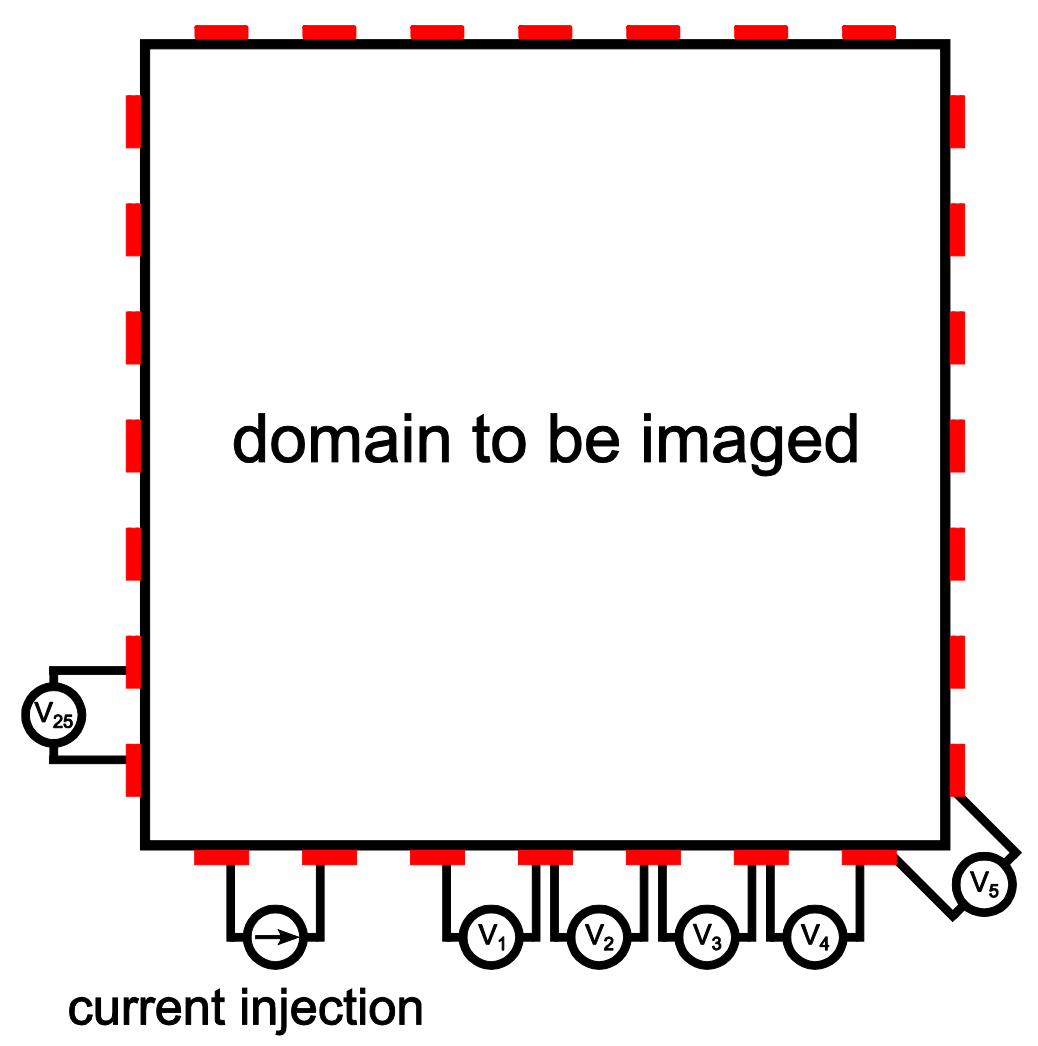

Figure 1. EIT injection schematic with 28 electrodes. Current is injected between the first electrode pair while voltages are measured between electrode pairs not actively involved in the current injection. The current source is then moved about the domain such that every electrode pair receives a current injection.

\subsection{Forward Problem}

The EIT forward problem is the process of analytically simulating the previously described current injections. Laplace's equation governs the relationship between current and voltages for steady-state diffusion. In the absence of internal sources, this can be expressed as shown in equation (1).

$$
\boldsymbol{\nabla} \cdot \sigma \boldsymbol{\nabla} \phi=0
$$

Here, $\sigma$ is the conductivity distribution and $\phi$ is the domain potential. This partial differential equation is subject to the complete electrode model boundary condition that assumes the voltage on each electrode is constant (i.e. the electrodes are perfect conductors) and there is a voltage 
drop between the electrodes and the domain due to imperfect contact or contact impedance. This is expressed in equation (2). Equation (3) enforces the conservation of charge by requiring that the current through all of the electrodes sums to zero.

$$
\begin{gathered}
\sigma \nabla \phi \cdot \boldsymbol{n}=\frac{1}{z_{l}}\left(V_{l}-\phi\right) \\
\sum_{l=1}^{L} \int_{E_{l}} \sigma \nabla \phi \cdot \boldsymbol{n} \mathrm{d} S_{l}=0
\end{gathered}
$$

In the preceding, $\boldsymbol{n}$ is an outward pointing normal vector, $V_{l}$ is the voltage on the lth electrode, $z_{l}$ is the contact impedance between the lth electrode and the domain, and $L$ is the total number of electrodes. These equations are most conveniently solved by the finite element method as shown in the following.

$$
\begin{gathered}
{\left[\begin{array}{cc}
\boldsymbol{A}_{M}+\boldsymbol{A}_{Z} & \boldsymbol{A}_{W} \\
\boldsymbol{A}_{W}^{T} & \boldsymbol{A}_{D}
\end{array}\right]\left[\begin{array}{l}
\boldsymbol{\Phi} \\
\boldsymbol{V}
\end{array}\right]=\left[\begin{array}{l}
\mathbf{0} \\
\boldsymbol{I}
\end{array}\right]} \\
A_{Z i j}=\sum_{l=1}^{L} \int_{E_{l}} \frac{1}{z_{l}} w_{i} w_{j} \mathrm{~d} S_{l} \\
A_{W l i}=-\int_{E_{l}} \frac{1}{z_{l}} w_{i} \mathrm{~d} S_{l} \\
A_{D}=\operatorname{diag}\left(\frac{E_{l}}{z_{l}}\right)
\end{gathered}
$$

$\boldsymbol{A}_{M}$ is the standard stiffness matrix for steady-state diffusion, $w_{i}$ is the ith finite element interpolation function, $\boldsymbol{\Phi}$ is a vector of the domain solution, $\boldsymbol{V}$ is a vector of the electrode solutions, and $\boldsymbol{I}$ is a vector of current injections. 


\subsection{Inverse Problem}

The EIT inverse problem minimizes the difference between a vector of experimentally collected boundary voltages and boundary voltage predictions provided by the forward operator. This minimization is shown in equation (8).

$$
\boldsymbol{\sigma}^{*}=\arg \min _{\boldsymbol{\sigma}}\left(\left\|\boldsymbol{V}_{m}-\boldsymbol{F}(\boldsymbol{\sigma})\right\|^{2}\right)
$$

$\boldsymbol{V}_{m}$ is a vector of the experimentally collected boundary voltages, $\boldsymbol{F}(\boldsymbol{\sigma})$ is a vector of boundary voltage predictions provided by the forward problem, and $\boldsymbol{\sigma}^{*}$ is a conductivity distribution that satisfies the minimization. Note that the conductivity distribution has been boldfaced to reflect that it too is a vector due to the finite element discretization.

Next, $\boldsymbol{F}(\boldsymbol{\sigma})$ is linearized by performing a Taylor series expansion about some initial conductivity estimate, $\boldsymbol{\sigma}_{0}$, and retaining only the linear terms as shown in equation (9). This approximation is then substituted into equation (8) resulting in equation (10).

$$
\begin{gathered}
\boldsymbol{F}(\boldsymbol{\sigma}) \approx \boldsymbol{F}\left(\boldsymbol{\sigma}_{0}\right)+\frac{\partial \boldsymbol{F}\left(\boldsymbol{\sigma}_{0}\right)}{\partial \boldsymbol{\sigma}}\left(\boldsymbol{\sigma}-\boldsymbol{\sigma}_{0}\right) \\
\boldsymbol{\sigma}^{*}=\arg \min _{\boldsymbol{\sigma}}\left(\left\|\boldsymbol{V}_{m}-\boldsymbol{F}\left(\boldsymbol{\sigma}_{0}\right)-\frac{\partial \boldsymbol{F}\left(\boldsymbol{\sigma}_{0}\right)}{\partial \boldsymbol{\sigma}}\left(\boldsymbol{\sigma}-\boldsymbol{\sigma}_{0}\right)\right\|^{2}\right)
\end{gathered}
$$

By substituting $\boldsymbol{V}_{e}=\boldsymbol{V}_{m}-\boldsymbol{F}\left(\boldsymbol{\sigma}_{0}\right), \boldsymbol{J}=\partial \boldsymbol{F}\left(\boldsymbol{\sigma}_{0}\right) / \partial \boldsymbol{\sigma}$, and $\Delta \boldsymbol{\sigma}=\boldsymbol{\sigma}-\boldsymbol{\sigma}_{0}$, equation (10) can be restated as equation (11).

$$
\boldsymbol{\sigma}^{*}=\arg \min _{\boldsymbol{\sigma}}\left\|\boldsymbol{V}_{e}-\boldsymbol{J} \Delta \boldsymbol{\sigma}\right\|^{2}
$$

Recovering $\Delta \boldsymbol{\sigma}$ from equation (11), however, requires an additional consideration because $\boldsymbol{J}$ is severely rank-deficient. Therefore, Tikhonov regularization is employed such that the explicit solution to $\Delta \boldsymbol{\sigma}$ can be expressed as shown in equation (12). 


$$
\Delta \boldsymbol{\sigma}=\left(\boldsymbol{J}^{T} \boldsymbol{J}+\alpha^{2} \boldsymbol{L}^{T} \boldsymbol{L}\right)^{-1} \boldsymbol{J}^{T} \boldsymbol{V}_{\boldsymbol{e}}
$$

Here, $\boldsymbol{L}$ is a regularization term and its contribution is controlled by $\alpha$. The unweighted discrete Laplace operator is herein employed for regularization. The conductivity distribution is then updated iteratively as $\boldsymbol{\sigma}_{n+1}^{*}=\boldsymbol{\sigma}_{n}^{*}+\Delta \boldsymbol{\sigma}$ until the error is sufficiently minimized.

Lastly, $\boldsymbol{J}$, also known as the sensitivity matrix, can be formed by enforcing the conservation of power through the electrodes and in the domain thereby relating electrode voltage perturbations to conductivity perturbations as shown in equation (13) [32].

$$
J_{M N e}=-\int_{\Omega_{e}} \nabla \phi^{M} \cdot \nabla \bar{\phi}^{N} \mathrm{~d} \Omega_{e}
$$

Computationally, $M N$ is a single index of $\boldsymbol{J}$ and refers to the integral of the contraction of the gradient of the voltage on the eth element due to current supplied by the $M$ th electrode injection pair and the gradient of the voltage on the eth element due to the $N$ th adjoint field. The adjoint field is the domain solution due to a unit current injection being supplied to the $N$ th electrode measurement pair. This integral is evaluated over the eth element. This can also be thought of as the sensitivity of the $N$ th electrode measurement pair due to a slight conductivity perturbation of the eth finite element when the current is injected in the Mthelectrode pair. 


\section{Experimental}

\subsection{CNF/PU Manufacturing}

CNFs are hollow fibers comprised of stacked cone-like carbon structures with a length-to-outerdiameter ratio ranging between 250 and 2000 [33]. They are less costly than carbon nanotubes (CNTs) while retaining acceptable mechanical (elastic modulus of $240 \mathrm{GPa}$ and tensile strength of $2.92 \mathrm{GPa}$ [34]), electrical (electrical conductivity of $105 \mathrm{~S} \mathrm{~m}^{-1}$ [35]), and thermal properties (thermal conductivity of $1950 \mathrm{~W} \mathrm{~m}^{-1} \mathrm{~K}^{-1}[36]$ ). Because CNFs cost less than CNTs while retaining good mechanical and transport properties, they are attractive from a manufacturing perspective. Four CNF/PU specimens were manufactured using ReoFlex 20 PU from Smooth-On and Pyrograf III-PR-24-XT-HHT CNFs from Applied Sciences. Measured amounts of polyurethane and CNFs were combined to produce specimens at 7.5, 10.5, 12.5, and 15\% filler volume fraction. CNFs and polyurethane were first hand-mixed in a cup to form a dough-like material. This mixture was then placed into a 2-roll mill and shear-mixed. Specimens containing 10.5, 12.5, and $15 \% \mathrm{CNF}$ filler were shear-mixed for 5 minutes, whereas the specimen containing 7.5\% CNF was shear-mixed for 20 minutes in order to obtain good dispersion and similar CNF aspect ratio. Immediately after mixing, the material was spread evenly into an open, rectangular silicone mold measuring $25.4 \mathrm{~mm} \times 76.2 \mathrm{~mm} \times 2.5 \mathrm{~mm}$. A flat cover was then put onto the top of the mold and the material was cured at room temperature for at least 16 hours. Lastly, the material was post-cured for an additional 6 hours at $65^{\circ} \mathrm{C}$ to stabilize the mechanical properties of the polyurethane. 

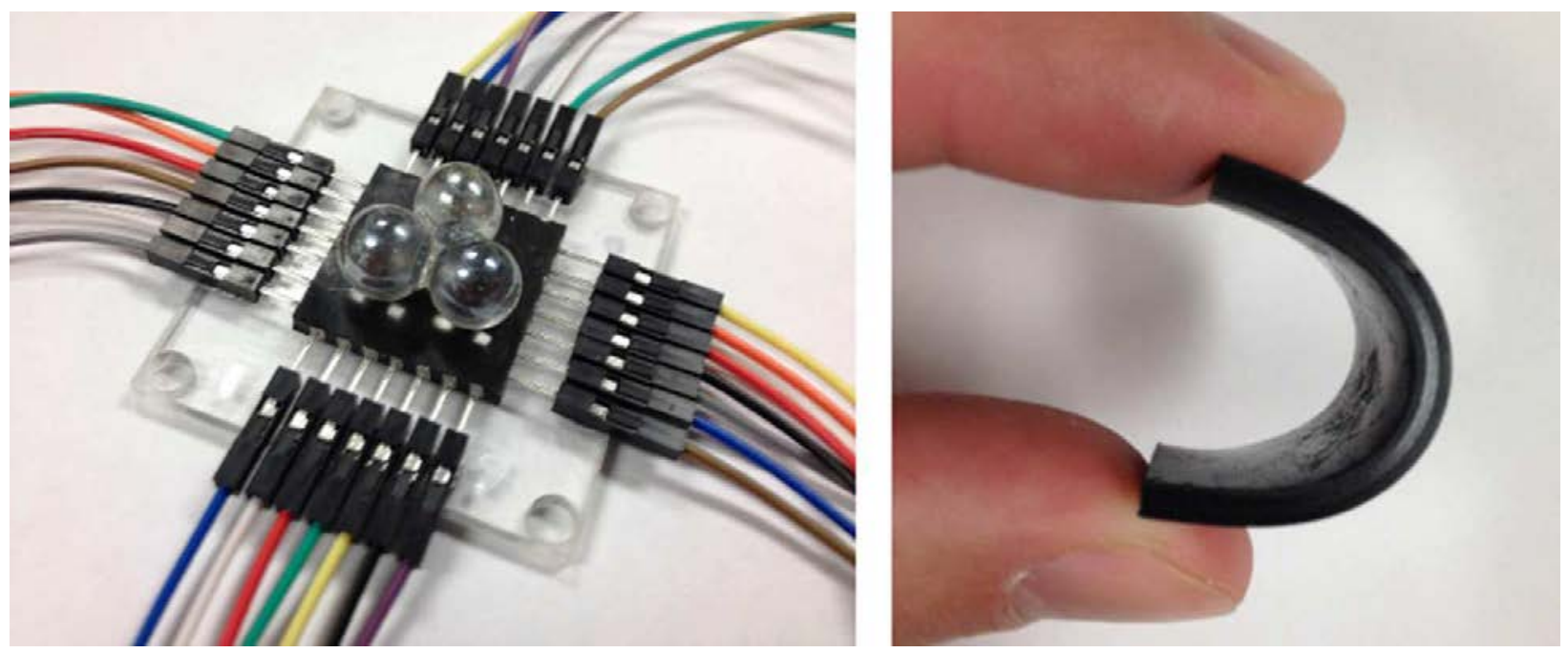

Figure 2. Left: EIT set up with marble indenters atop the CNF/PU nanocomposite. Electrodes made of patches of colloidal silver paste can be seen on the edges of the composite. Right: demonstration of the flexibility of the CNF/PU nanocomposite. The material is easily deformed and recovers to its original state.

Post-dispersion CNFs retain, on average, a diameter of $0.1 \mu \mathrm{m}$ and a length of $11.5 \mu \mathrm{m}$. As shown in Figure 2, these nanocomposites are highly flexible and deformable by hand. The manufacturer's data states that the polyurethane has a Shore hardness of approximately $20 \mathrm{~A}$ and a tensile modulus of approximately $190 \mathrm{MPa}$ at $100 \%$ strain. The influence of filler volume fraction on microscale morphology can be seen from the SEM images in Figure 3. Figure 4 shows a close up of a single CNF protruding from the PU and multiple CNFs protruding from a fracture surface. These images provide a qualitative assessment that the fillers are not aggregated.

SEM imaging was performed via a Hitachi SU8000 In-Line SEM in the Lurie Nanofabrication Center at the University of Michigan. CNF/PU specimens were immersed in liquid nitrogen for approximately 10 minutes and then fractured in order to obtain a surface for imaging. 

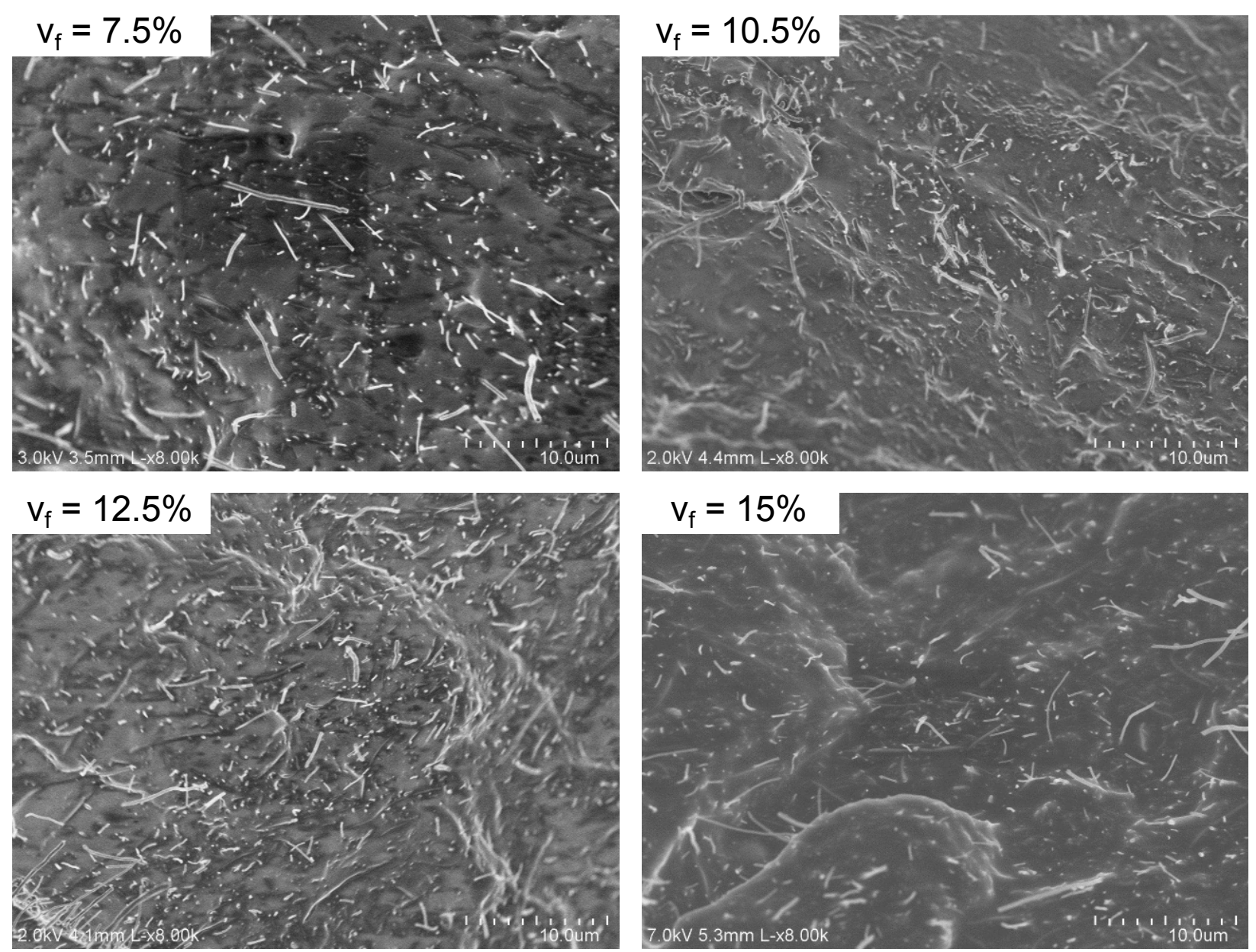

Figure 3. SEM images of CNF distribution at $7.5 \%$ filler volume fraction, $10.5 \%$ filler volume fraction, $12.5 \%$ filler volume fraction, and $15 \%$ filler volume fraction. 

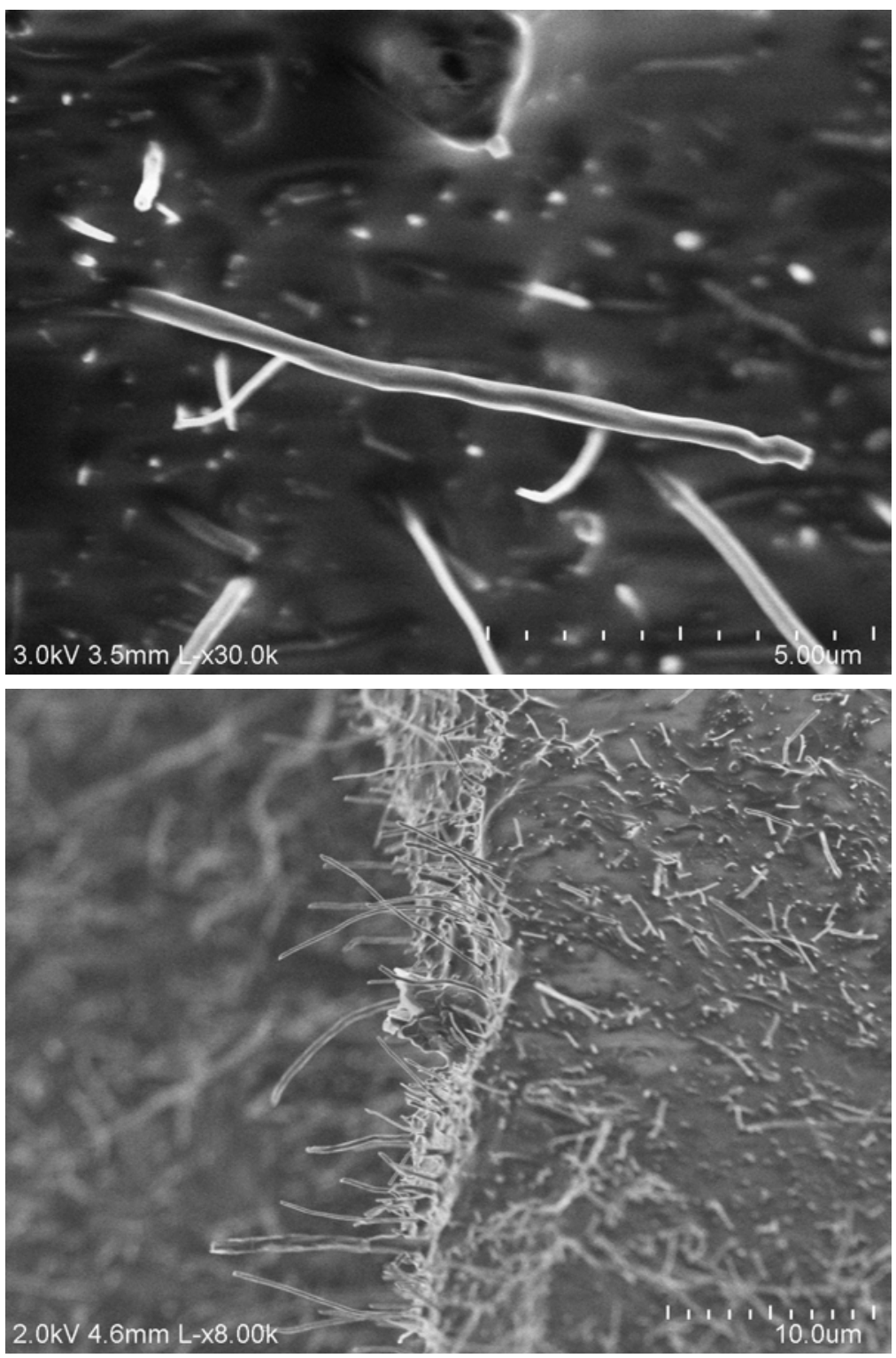

Figure 4. Top: close up of single CNF from 7.5\% filler volume fraction sample. Bottom: CNFs protruding from fracture surface in $12.5 \%$ filler volume fraction sample.

\subsection{Bonded Substrate Testing}

Tests of the CNF/PU nanocomposites were performed in order to assess the influence of filler volume fraction and viscoelasticity on piezoresistive response when bonded to a much stiffer substrate undergoing mechanical loading. These tests are important for a couple of reasons. First, because the PU is a viscoelastic material, its strain state relaxes considerably over time. Because nanocomposite conductivity is a function of the strain state, its conductivity will also evolve over 
time, and it is therefore important to have some insight into the rate at which the conductivity will equilibrate. Second, there is a growing interest in using nanocomposites as discrete strain sensors with higher gauge factors than traditional strain gauges [7] [8]. In these applications as discrete strain sensor, nanocomposites will necessarily be bonded to much stiffer substrates.

To this end, thin strips of the CNF/PU specimens measuring $25.4 \mathrm{~mm} \times 6.35 \mathrm{~mm} \times 2.5 \mathrm{~mm}$ were completely bonded onto polyvinyl chloride (PVC) bars, loaded into a MTS-810 load frame, and strained at $0.1 \mathrm{~mm} / \mathrm{s}$. Colloidal silver paste (TedPella 16032) electrodes were applied such that the resistance of the material could be measured along the $25.4 \mathrm{~mm}$ dimension via an Agilent 34401A digital multimeter. The longitudinal strain of the PVC bars was measured via a strain gauge bonded to the back surface of the PVC bars. Two loading regimens were considered. In the first, the PVC bar was subjected to linearly increasing tension, sinusoidal variation between tension and compression, and finally linearly decreasing compression. The second load regimen applied and held constant tension intermittently. To provide some insight into the repeatability of the material, each specimen was subjected to the previously described loadings twice with one day elapsing between tests. The longitudinal strain of the PVC bars as measured by the attached strain gauges and the percent change in resistance of the CNF/PU specimens can be seen in Figures $5-8$ where the red lines correspond to the resistance change of the first loading and the red dots correspond to the second loading.

\subsection{Electrical Impedance Tomography Setup}

Distributed strain was induced in $25.4 \mathrm{~mm} \times 25.4 \mathrm{~mm} \mathrm{CNF} / \mathrm{PU}$ samples by resting a $1.2 \mathrm{~kg}$ mass atop three bonded glass marbles as shown in Figure 1. Spherical glass marbles were used because they are non-conductive and their curvature does not cut into the soft CNF/PU. Three marbles were used to demonstrate the ability of EIT to clearly differentiate between multiple points of contact. In light of the viscoelastic effects observed during tensile testing, the mass was allowed to rest atop the $\mathrm{CNF} / \mathrm{PU}$ samples for an hour before using a 28-electrode system to image strain-induced conductivity changes with an in-house EIT routine. 
Electrodes were attached to the CNF/PU composites by first applying seven evenly spaced 1.59 $\mathrm{mm}$ patches of colloidal silver paste per side as shown in Figure 2. The paste was allowed to dry for at least one hour before jumper wires were pushed lightly into the patches and then glued to an acrylic (PMMA) base. An additional drop of silver paste was then applied where the jumper wire touched the originally applied patch to ensure good electrical contact. A Keithley 6221 current source was used to supply $2.5 \mathrm{~mA}$ DC injections between electrodes. Voltages were measured using two 16-channel National Instruments 6368-PXIe data acquisition cards for $10 \mathrm{~s}$ at $128 \mathrm{~Hz}$. Data was collected via an in-house LabView code and smoothed using a moving average of half-width 256. It is also important to note that the boundary voltages collected over the $10 \mathrm{~s}$ period were constant indicating that all viscoelastic effects had dissipated.

\section{Results and Discussion}

\subsection{Bonded Substrate Piezoresistive Response}

Consider first the results for the sinusoidally varying strains as shown tothe left of Figures $5-8$. For positive tensile strain, the resistance of the $7.5 \%$ filler volume fraction material first increases with increasing tension. As the strain cycle goes into compression the nature of thepiezoresistive response changes such that resistance again increases with increasing compression. A similar albeit muted trend is observed for $10.5 \%$. These results also resemble observations by Dubey et al. [37]. However, at 12.5 and $15 \%$ filler volume fraction the piezoresistive response of the material changes markedly to the point of appearing out of phase with the strain. While a complete analysis of the nature of the mechanisms of positive and negative piezoresistivity (i.e. positive or negative gauge factor) exceeds the scope of this study, Cattin and Hubert [38] and Toprakci et al. [39] provide excellent insight into the topic and interested readers are directed to their preceding citations for more thorough consideration.Quite succinctly, the change in piezoresistive response can be attributed to the high aspect ratio of the fillers. That is, for sufficiently small strains the nanofillers undergo relatively little rotation and filler-to-filler spacing increases or decreaseson average with tension or compression respectively.Beyond small strains, however, rotations of the high aspect ratio fillers can cause them to form or eliminate tunneling junctions that dominate changes in the average filler-to-filler 
spacing resulting in complex piezoresistive responses including changes in gauge factor sign. It is apparent that the dominant mechanism of piezoresistivity in $\mathrm{CNF} / \mathrm{PU}$ is volume fractiondependent and changes markedly between 10.5 and $12.5 \%$ filler volume fraction.

Now consider the right-most images of Figures $5-8$ wherein the PVC bars were put in tension, held, and then released for several cycles. All volume fractions respond immediately to the sudden application of tensile strain. However, the most dramatic change in resistance occurs either with the application of tension to the PVC bar or the release of tension with decay occurring between due to the viscoelastic response of the PU. The influence of a suddenly released load dominates the response for 12.5 and $15 \%$ filler volume fraction again suggesting a volume fraction-dependent dominant mechanism of piezoresistivity. Interested readers are directed to Dubey et al. [37] for a discussion on the influence of strain rate on piezoresistive response. It is also important to note that because of shear lag in the viscoelastic CNF/PU strip, determination of the strain state of the $\mathrm{CNF} / \mathrm{PU}$ is not trivial. It is stress free on all sides except where bonded, and the traction on the bonded side is dictated by the response of the PVC bar which, for regions sufficiently removed from the grips, is in a state of pure uniaxial stress. We reiterate, however, that highly compliant nanocomposite transducers bonded to structures for strain sensing will encounter a similar situation. It is therefore important to conduct tests as we have described before employing such nanocomposites as discrete, strain-sensing transducers.We also note that the piezoresistive response for each filler volume fraction follows similar trends between the two loadings with only minor changes in magnitude as indicated by the similarity of the solid red lines and the red dots in Figures $5-8$. 

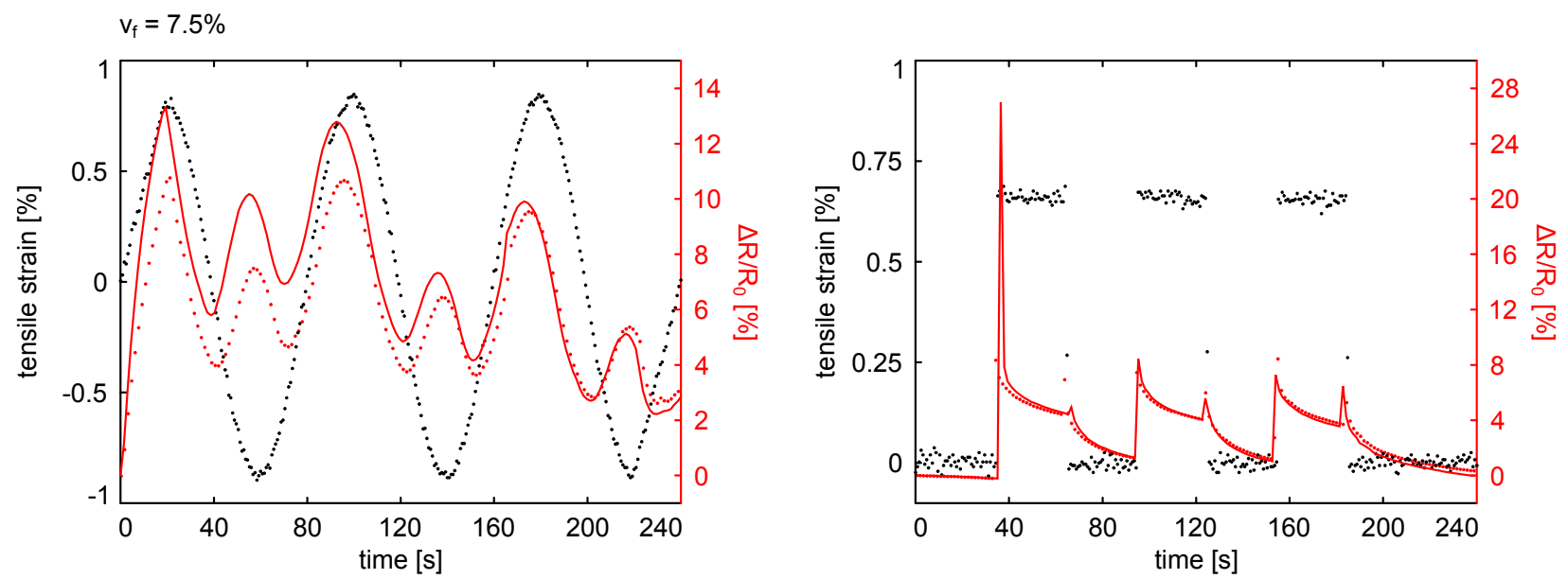

Figure 5. Percent change in resistance during mechanical loading of bonded CNF/PU material with $7.5 \%$ filler volume fraction. The left image shows the response to linearly increasing tension, sinusoidally varying tension and compression, and, lastly, linearly decreasing compression. The right image shows the response to cyclically applied and held tension. The red lines correspond to the resistance change during the first test while the red dots correspond to the resistance change during the second test. The applied strain was the same in each test.
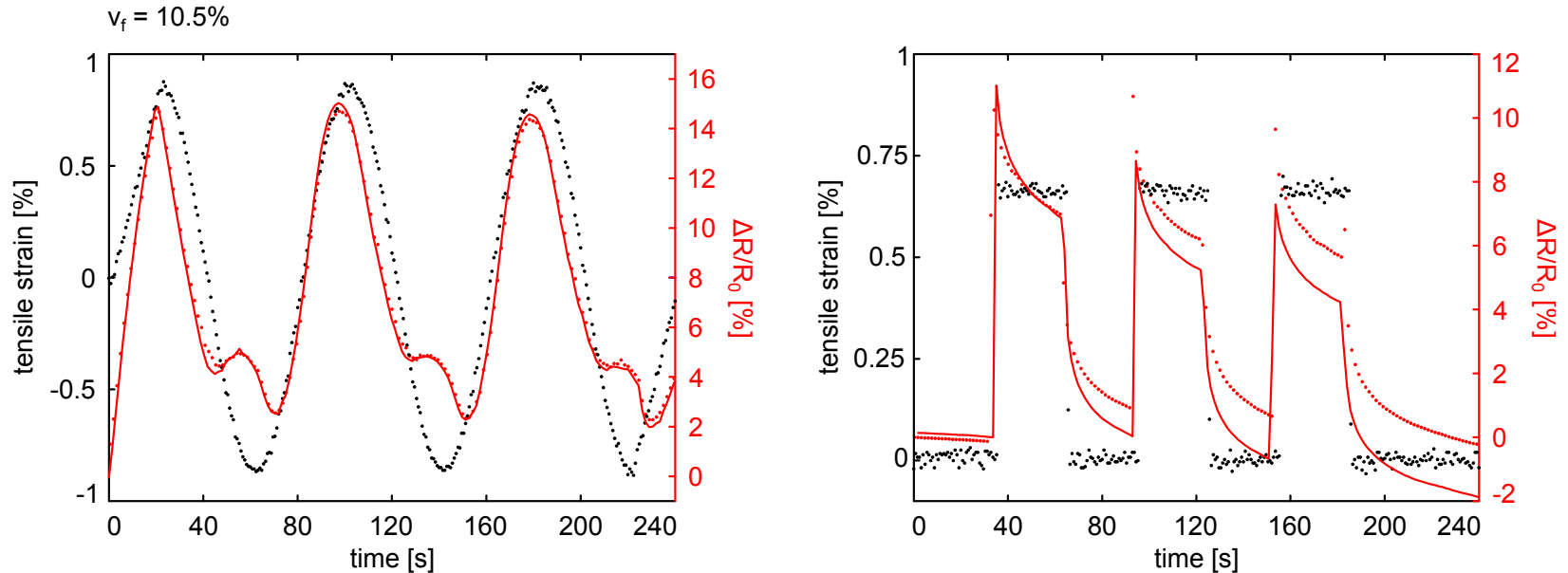

Figure 6. Percent change in resistance during mechanical loading of bonded CNF/PU material with $10.5 \%$ filler volume fraction. 

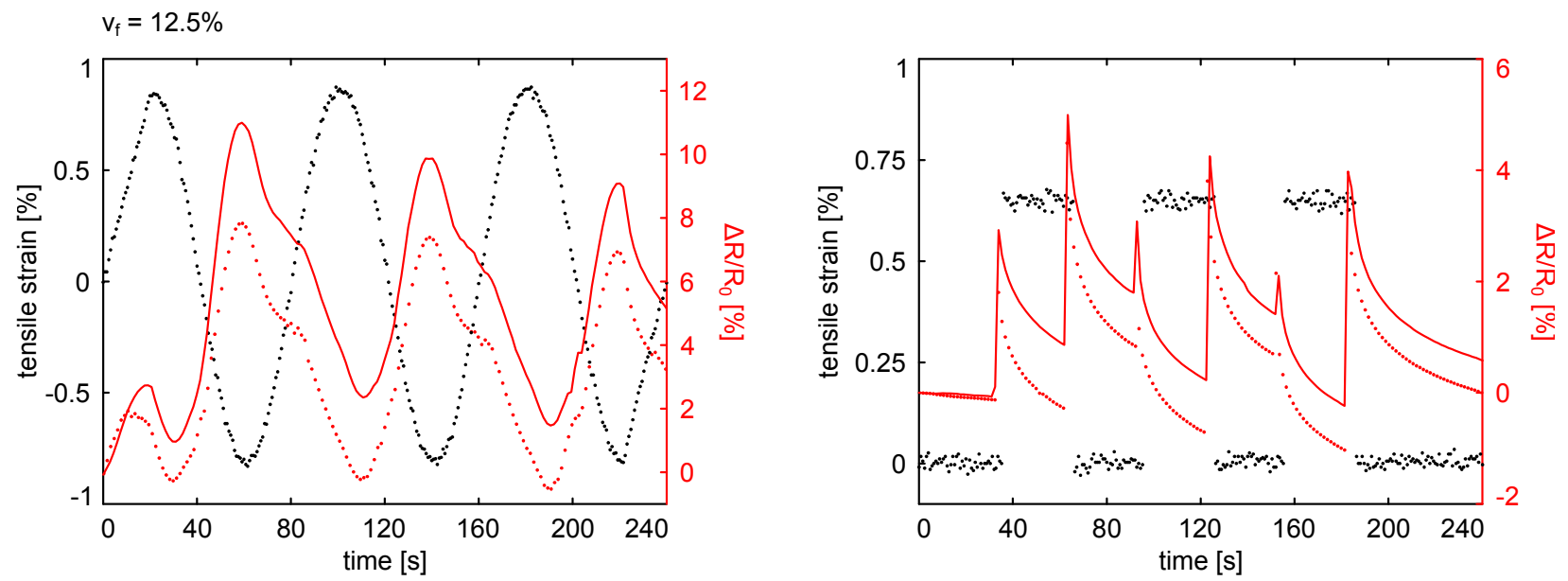

Figure 7. Percent change in resistance during mechanical loading of bonded CNF/PU material with $12.5 \%$ filler volume fraction.
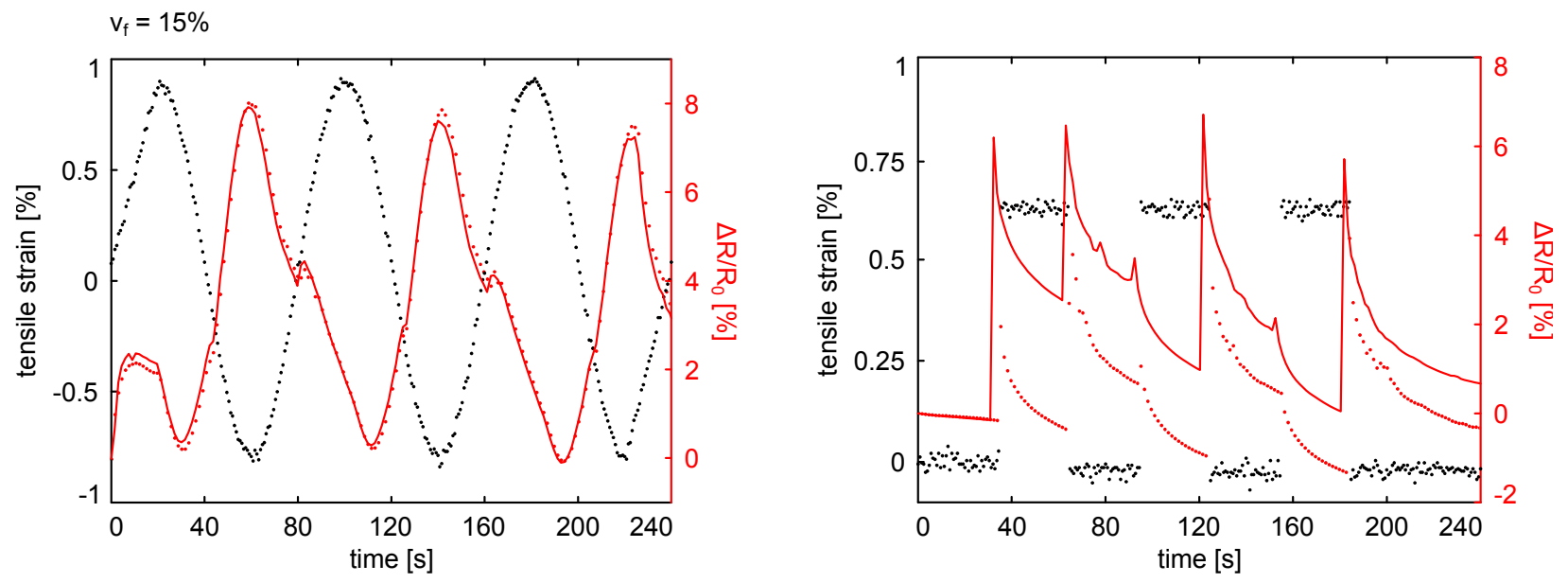

Figure 8. Percent change in resistance during mechanical loading of bonded CNF/PU material with $15 \%$ filler volume fraction.

\subsection{Distributed Strain Sensing}

As shown in Figure 9, EIT accurately captures three distinct points of contact due to the spherical indenters for each volume fraction. Larger changes in conductivity are observed for lower volume fractions implying that they are more sensitive to the imposed strain fields. However, the EIT image produced for $7.5 \%$ filler volume fraction has a region in which the conductivity change is markedly larger. This is speculated to be due to non-uniform nanofiller dispersion and a region of lower nanofiller density in the vicinity of the region in question. 
Conversely, as the filler volume fraction increases, such deviations are less pronounced. Contrasting the results shown in Figures 5-8 with the results shown in Figure 9, it can be inferred that the type of loading markedly influences the piezoresistive response of CNF/PU. That is, conductivity increases were seen for all filler volume fractions through EIT imaging wherein Hertzian contact-like strains were induced. The CNF/PU, however, displayed greatly varying piezoresistivity when bonded to a stiffer substrate in dynamic sinusoidal loading. Conductivity increases in the region of the distributed load indicating that the nanofillers are becoming closer together thereby decreasing the tunneling resistance felt by electrons. Additionally, the compression increases the density of the nanofiller network thereby increasing the number of viable tunneling junctions. These factors seemingly dominate the response resulting in a net increase in conductivity. 


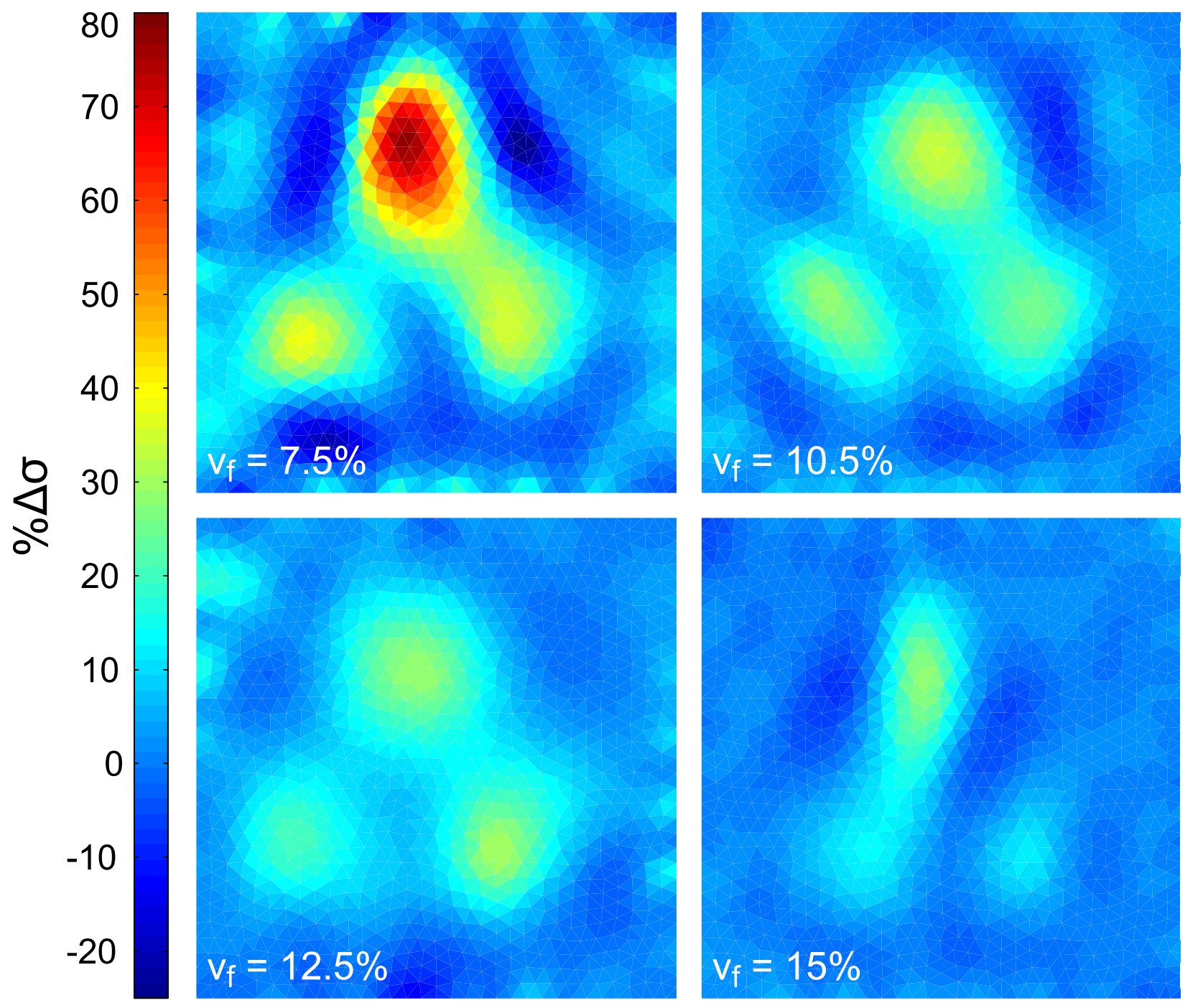

Figure 9. EIT images of percent change in conductivity due to distributed straining. EIT clearly captures three distinct points of contact, and larger increases in conductivity are observed for lower volume fractions.

In order to further elucidate the potential of $\mathrm{CNF} / \mathrm{PU}$ for tactile sensing, additional EIT testing was conducted on the $10.5 \%$ filler volume fraction specimen. This particular specimen was selected because of its repeatability in tensile testing in Figure 6 and spatial uniformity in Figure 9. Here, four marbles were arranged in a square at the center of the CNF/PU specimen. The same $1.2 \mathrm{~kg}$ mass was applied for an hour. The $10.5 \%$ filler volume fraction specimen was subjected to the same load twice and imaged twice with one day between tests. The resulting EIT images can be seen in Figure 10. 


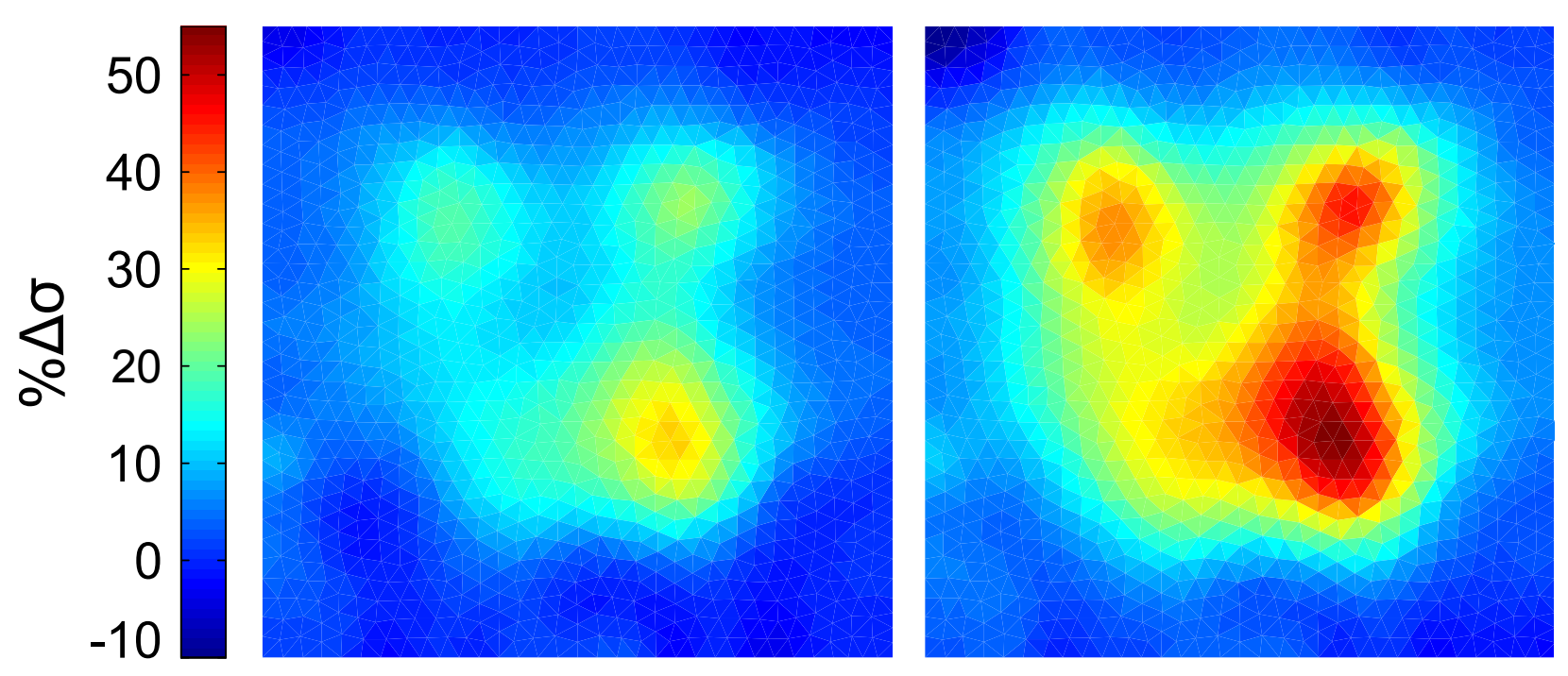

Figure 10. $10.5 \%$ filler volume fraction specimen imaged with four points of contact. Left: initial image. Right: second image produced one day later on the same specimen subjected to the same loading.

As seen in Figure 10, EIT does indeed capture four points of contact (albeit the lower-left point is much less pronounced); furthermore, both the left and right images have very similar shapes. This indicates that the method of tactile sensing herein described does have good spatial repeatability. However, a non-negligible difference in magnitude of conductivity change exists indicating that the material is less capable of consistently producing the same change in conductivity for a given load. This is also observed in Figures 5-8; the piezoresistive response between tests conducted on the same material a day apart produces similar trends but with different magnitudes.

\section{Summary and Conclusions}

We have herein investigated employing CNF/PU nanocomposites and EIT for tactile imaging and distributed strain sensing. Regions imaged via SEM show no aggregation. Tests of the $\mathrm{CNF} / \mathrm{PU}$ nanocomposite material bonded to a mechanically deformed substrate revealed that the piezoresistive response depends strongly on the nanofiller volume fraction. Furthermore, the material displayed a time-dependent response. Distributed strain sensing via EIT, however, resulted in much more consistent results. Three distinct points of contact were imaged via EIT, 
and sensitivity increased with decreasing filler volume fraction. The relative ease and low cost of $\mathrm{CNF} / \mathrm{PU}$ manufacturing combined with our results demonstrate the considerable potential of CNF/PU and EIT for tactile imaging and distributed strain sensing. However, because of the complicated volume fraction and time-dependent piezoresistive responses observed, future work should more exhaustively explore and characterize the mechanisms of these responses prior to employing CNF/PU in real applications. Furthermore, future work should examine the repeatability of the performance of CNF/PU sensors in a statistically significant way and endeavor to find the lowest filler volume fraction that will provide uniform spatialsensitivityand a reliably repeatable piezoresistive response.Despite these key practical challenges, our results demonstrate the considerable potential of the approach outlined herein.

\section{Acknowledgements}

This research is partially supported by the US Army Research Office (W911NF-10-1-00267) and the National Science Foundation (CMMI-DS-1232436).

The authors gratefully thank Dr. Pilar Herrara-Fiero for performing the SEM imaging.

The authors gratefully thank Professor Jerome P. Lynch and his graduate student research assistant Andrew R. Burton for assisting in the tensile testing experiments and Professor Victor Li for the use of his MTS-810 load frame.

\section{References}

[1] Tee BCK, Wang C, Allen R, Bao Z. 2012 An electrically and mechanically self-healing composite with pressure- and flexion-sensitive properties for electronic skin applications. Nature Nanotechnology. 7: 825-832.

[2] Lipomi DJ, Vosgueritchian M, Tee BCK, Hellstrom SL, Lee JA, Fox CH, et al. 2011 Skinlike pressure and strain sensors based on transparent elastic films of carbon nanotubes. Nature Nanotechnology. 6: 788-792.

[3] Mannsfeld SCB, Tee BCK, Stoltenberg RM, Chen CVHH, Barman S, Muir BVO, et al. 
2010 Highly sensitive flexible pressure sensors with microstructured rubber dielectric layers. Nature Materials. 9: 859-864.

[4] Simmons JG. 1963 Generalized formula for the electric tunnel effect between similar electrodes separated by a thin insulating film. Journal of Applied Physics. 34: 1793.

[5] Hu N, Masuda Z, Yamamoto G, Fukunaga H, Hashida T, Qiu J. 2008 Effect of fabrication process on electrical properties of polymer/multi-wall carbon nanotube nanocomposites. Composites Part A: Applied Science and Manufacturing. 39: 893-903.

[6] Hu N, Karube Y, Yan C, Masuda Z, Fukunaga H. 2008 Tunneling effect in a polymer/carbon nanotube nanocomposite strain sensor. Acta Materialia. 56: 2929-2936.

[7] Hu N, Karube Y, Arai M, Watanabe T, Yan C, Li Y, et al. 2010 Investigation on sensitivity of a polymer/carbon nanotube composite strain sensor. Carbon. 48: 680-687.

[8] Kang I, Schulz MJ, Kim JH, Shanov V, Shi D. 2006 A carbon nanotube strain sensor for structural health monitoring. Smart Materials and Structures. 15: 737.

[9] Loh KJ, Kim J, Lynch JP, Kam NWS, Kotov NA. 2007 Multifunctional layer-by-layer carbon nanotube-polyelectrolyte thin films for strain and corrosion sensing. Smart Materials and Structures. 16: 429-438.

[10] Loh KJ, Lynch JP, Shim BS, Kotov NA. 2008 Tailoring piezoresistive sensitivity of multilayer carbon nanotube composite strain sensors. Journal of Intelligent Material Systems and Structures. 19: 747-764.

[11] Tallman T, Wang KW. 2013 An arbitrary strains carbon nanotube composite piezoresistivity model for finite element integration. Applied Physics Letters. 102: 011909.

[12] Gao L, Chou TW, Thostenson ET, Zhang Z. 2010 A comparative study of damage sensing in fiber composites using uniformly and non-uniformly dispersed carbon nanotubes. Carbon. 48: 3788-3794.

[13] Gao L, Chou TW, Thostenson ET, Zhang Z, Coulaud M. 2011 In situ sensing of impact damage in epoxy/glass fiber composites using percolating carbon nanotube networks. Carbon. 49: 3382-3385.

[14] Kim KJ, Yu WR, Lee JS, Gao L, Thostenson ET, Chou TW, et al. 2010 Damage characterization of 3D braided composites using carbon nanotube-based in situ sensing. 
Composites: Part A. 41: 1531-1537.

[15] Thostenson ET, Chou TW. 2006 Carbon Nanotube Networks: Sensing of Distributed Strain and Damage for Life Prediction and Self Healing. Advanced Materials. 18: 2837-2841.

[16] Wu AS, Coppola AM, Sinnott MJ, Chou TW, Thostenson ET, Byun JH, et al. 2012 Sensing of damage and healing in three-dimensional braided composites with vascular channels. Composites Science and Technology. 72: 1618-1626.

[17] Naghashpour A, Hoa SV. 2013 A technique for real-time detection, location and quantification of damage in large polymer composite structures made of electrically nonconductive fibers and carbon nanotube networks. Nanotechnology. 24: 455502.

[18] Naghashpour A, Hoa SV. 2015 A technique for real-time detecting, locating, and quantifying damage in large polymer composite structures made of carbon fibers and carbon nanotube networks. Structural Health Monitoring. 14: 35-45.

[19] Zhang D, Ye L, Wang D, Tang Y, Mustapha S, Chen Y. 2012 Assessment of transverse impact damage in GF/EP laminates of conductive nanoparticles using electrical resistive tomography. Composites: Part A. 43: 1587-1598.

[20] Viets C, Kaysser S, Schulte K. 2014 Damage mapping of GFRP via electrical resistance measurements using nanocomposite epoxy matrix systems. Composites: Part B. 65: 80-88.

[21] Hou TC, Loh KJ, Lynch JP. 2007 Spatial conductivity mapping of carbon nanotube composite thin films by electrical impedance tomography for sensing applications. Nanotechnology. 18: 315501.

[22] Loh KJ, Hou TC, Lynch JP, Kotov NA. 2009 Carbon nanotube sensing skins for spatial strain and impact damage identification. Journal of Nondestructive Evaluation. 28: 9-25.

[23] Loyola BR, Briggs TM, Arronche L, Loh KJ, La Saponara V, O'Bryan G, et al. 2013 Detection of spatially distributed damage in fiber-reinforced polymer composites. Structural Health Monitoring. 12: 225-239.

[24] Tallman TN, Gungor S, Wang KW, Bakis CE. 2014 Damage detection and conductivity evolution in carbon nanofiber epoxy via electrical impedance tomography. Smart Materials and Structures. 23: 045034.

[25] Tallman TN, Gungor S, Wang KW,BCE. 2015 Damage Detection via Electrical Impedance 
Tomography in Glass Fiber/Epoxy Laminates with Carbon Black Filler. Structural Health Monitoring. 14: 100-109.

[26] Kim B, Lu Y, Kim T, Han JW, Meyyappan M, Li J. 2014 Carbon Nanotube Coated Paper Sensors for Damage Diagnosis. ACS Nano. 8: 12092-12097.

[27] Ferreira A, Cardoso P, Klosterman D, Covas JA, van Hattum FWJ, Vaz F, et al. 2012 Effect of filler dispersion on the electromechanical response of epoxy/vapor-grown carbon nanofiber composites. Smart Materials and Structures. 21: 075008.

[28] Yasuoka T, Shimamura Y, Todoroki A. 2013 Patch-type large strain sensor using elastomeric composite filled with carbon nanofibers. International Journal of Aeronautical and Space Science. 14: 146-151.

[29] Silvera-Tawil D, Rye D, Soleimani M, Velonaki M. 2015 Electrical Impedance Tomography for Artificial Sensitive Robotic Skin: A Review. IEEE Sensors. 15: 20012016.

[30] Yao A, Soleimani M. 2012 A pressure mapping imaging device based on electrical impedance tomography of conductive fabrics. Sensor Review. 32: 310-317.

[31] Yao A, Yang CL, Seo JK, Soleimani M. 2013 EIT-Based Fabric Pressure Sensing. Computational and Mathematical Methods in Medicine. 2013: 405325.

[32] Holder DS, editor. 2005 Electrical Impedance Tomography: Methods, History and Applications. (Bristol: Institute of Physics).

[33] Al-Saleh MH, Sundararaj U. 2011 Review of the mechanical properties of carbon nanofiber/polymer composites. Composites: Part A. 42: 2871-2880.

[34] Tibbetts GG, McHugh JJ. 1999 Mechanical properties of vapor-grown carbon fiber composites with thermoplastic matrices. Journal of Materials Research. 14: 2871-2880.

[35] Bal S. 2010 Experimental study of mechanical and electrical properties of carbon nanofiber/epoxy composites. Materials and Design. 31: 2406-2413.

[36] Lozano K, Barrera EV. 2001 Nanofiber-reinforced thermoplastic composites. I. Thermoanalytical and mechanical analysis. Journal of Applied Polymer Science. 79: 125133.

[37] Dubey KA, Mondal RK, Grover V, Bhardwaj YK, Tyagi AK. 2015 Development of a novel 
strain sensor based on fluorocarbon-elastomeric nanocomposites: Effect of network density on the electromechanical properties. Sensors and Actuators A: Physical. 221: 33-40.

[38] Cattin C, Hubert P. 2014 Piezoresistivity in Polymer Nanocomposites with High Aspect Ratio Particles. Applied Materials \& Interfaces. 6: 1804-1811.

[39] Toprakci HAK, Kalanadhabhatla SK, Spontak RJ, Ghosh TK. 2013 Polymer Nanocomposites Containing Carbon Nanofibers as Soft Printable Sensors Exhibiting StrainReversible Piezoresistivity. Advanced Functional Materials. 23: 5536-5542. 